\title{
Diversidade de larvas de Odonata (Insecta) da Bacia do Rio Ibicuí, Rio Grande do Sul, Brasil
}

\author{
Diversity of larvae Odonata (Insecta) River Basin Ibicuí, \\ Rio Grande do Sul, Brazil \\ Nícolas de Souza Brandão de Figueiredo, Mateus Marques Pires, Rosemary Cristina Souza Davanso, \\ Carla Bender Kotzian
}

Universidade Federal de Santa Maria, UFSM, Santa Maria, RS, Brasil

\begin{abstract}
Resumo
A diversidade de larvas de odonata em sistemas lóticos de dois trechos (encosta e planície) da bacia do Rio Ibicuí, Rio Grande do Sul, Brasil, foi inventariada nos anos de 2009 e 2010. Foram coletados 964 espécimes pertencentes a 34 gêneros distribuídos em sete famílias. Libellulidae foi a família mais rica com 14 gêneros e Gomphidae foi a mais abundante com 410 espécimes coletados. Três gêneros (Agriogomphus, Cacoides e Mnesarete) são novos registros para o estado do Rio Grande do Sul. Os gêneros dominantes e mais frequentes foram Progomphus, Hetaerina e Argia. Não foi encontrada diferença significativa de riqueza de gêneros entre os dois trechos estudados da bacia, mas uma diferença de frequência de ocorrência de alguns gêneros foi registrada. Essa diferença deve-se à distinção de características do substrato entre os trechos, que promoveram uma predominância de gêneros da família Gomphidae, em especial no trecho arenoso da planície.
\end{abstract}

Palavras-chave: inventário, libélulas, Neotrópicos, riachos.

\begin{abstract}
An inventory of larval odonates in lotic systems from two courses (slope and lowland) in the Ibicuí River basin, Rio Grande do Sul, Brazil, was carried out in 2009 and 2010. In total, 964 specimens were sampled and classified in 34 genera and seven families. Libellulidae was the richest family with 14 genera and Gomphidae was the most abundant. Three new genera (Agriogomphus, Cacoides and Mnesarete) are new records for Rio Grande do Sul State. The dominant and more frequent genera were Progomphus, Hetaerina and Argia. There was no significant difference in genus richness between the two courses, but a difference in frequency of occurrence of some genera was recorded. This difference is due to the distinguishing characteristics of the substrate between sections, which promoted a predominance of the genera of the gomphid family, especially in the sandy stretch from the lowland course.
\end{abstract}

Keywords: inventory, dragonflies, Neotropics, streams. 


\section{Introdução}

Odonatas são insetos hemimetábolos cujas larvas são encontradas em ambientes de água doce. Estas apresentam uma ampla diversidade morfológica, o que permite ao grupo habitar os mais diversos microhabitats (Corbet, 1999). O estágio larval pode durar de algumas semanas a vários anos, e requer condições específicas para seu desenvolvimento e estabelecimento no ambiente (Carvalho \& Nessimian 1998). O estabelecimento das larvas é influenciado por diversos fatores, primariamente a natureza do corpo d'água (Carvalho \& Nessimian 1998). Em ambientes lóticos, fatores relacionados às bacias hidrográficas também são capazes de afetar o estabelecimento dessa fauna, como a composição do substrato (Assis et al. 2004) e características da paisagem adjacente (Samways \& Steytler 1996). Dessa forma, em função das distintas condições ambientais presentes em uma bacia, pode-se esperar que diferentes trechos de uma mesma bacia hidrográfica apresentem faunas distintas entre si. Não obstante, estudos sobre a distribuição espacial da ordem permanecem escassos, especialmente em áreas neotropicais.

A ordem Odonata está presente desde regiões temperadas a tropicais e possui, aproximadamente, 6000 espécies descritas no mundo, pertencentes a 600 gêneros, agrupados em 33 famílias de três subordens, Anisoptera, Anisozygoptera e Zygoptera (Kalkman et al. 2008). Na América do Sul são conhecidas 637 espécies de Anisoptera atribuídas a 88 gêneros e sete famílias, e 682 espécies de Zygoptera, classificadas em 92 gêneros e 11 famílias (Domínguez \& Fernández 2009). No Brasil, até o momento foram registradas aproximadamente 800 espécies distribuídas em 123 gêneros e 14 famílias (Souza et al. 2007). Entretanto, os trabalhos realizados no país abrangem apenas 29\% do território, o que evidencia uma grande escassez de dados (De Marco \& Vianna 2005). As regiões mais bem amostradas estão inseridas em clima subtropical ou tropical, como a Sudeste, seguida pela Norte que é mais estudada ao longo do Rio Amazonas, e pela Centro-Oeste (Dalzochio et al. 2011).

De acordo com os critérios da Lista Vermelha de Espécies Ameaçadas de Extinção da IUCN (2008), uma a cada dez espécies de Odonata está em perigo crítico de extinção, e 35\% das espécies possuem dados insuficientes de ocorrência (Clausnitzer et al. 2009). Dentre as maiores ameaças às comunidades de odonatas estão ações antrópicas, como a degradação de ambientes aquáticos através de poluição, agricultura, represamento e desvio de rios (Kalkman et al. 2008, Clausnitzer et al. 2009).

O Estado do Rio Grande do Sul (RS) apresenta características que devem favorecer a presença de uma odonatofauna diversificada e diferenciada da do restante do país, como uma rica malha hidrográfica e regiões de clima mais frio e temperado (Maluf 2000). Contudo, poucos inventários dedicados exclusivamente a odonatas foram feitos. Para adultos, foram realizados estudos na região dos municípios de Porto Alegre (Teixeira et al. 1971) e de Santa Maria (Costa et al. 1971), e para larvas, em diversas áreas úmidas do Estado (Maltchik et al. 2010). Esse conhecimento é insuficiente, principalmente quando se considera que em ambientes lóticos de regiões com clima temperado, comunidades de certos grupos de macroinvertebrados podem apresentar riqueza maior do que as de ambientes lóticos de regiões com clima tropical (e.g. McKie et al. 2005, Floss et al. 2012). O presente trabalho teve como objetivo realizar um levantamento da odonatofauna, em fase larval, presente na bacia do Rio Ibicuí, uma das maiores do RS, e analisar a existência de diferenças, quanto à riqueza e composição, entre as comunidades dos cursos de encosta (superior) e de planície (médio-inferior) desta bacia.

\section{Material e Métodos}

\section{1 Área de estudo}

A bacia do Rio Ibicuí está situada nas regiões Central e da Fronteira Oeste do RS (Figura 1), abrange uma área total de $36.397,69 \mathrm{~km}^{2}$, e ocupa duas regiões geomorfológicas: o Planalto Meridional e a Depressão Central (Vieira 1984, Quadros \& Pillar 2002). No Planalto a altitude atinge cotas de $500 \mathrm{~m}$, e na Depressão Central, cerca de 50 m (Pereira et al. 1989). A vegetação presente na região está inserida nos domínios dos biomas Mata Atlântica (Floresta Estacional Decidual) e Pampa (Savanas estépicas), com Áreas de Tensão Ecológica (Quadros \& Pillar 2002). O clima na região de estudo é do tipo Cfa de Köppen, com precipitação anual média variando entre 1.250 a $2.000 \mathrm{~mm}$, e temperatura média anual, entre 15 e $18^{\circ} \mathrm{C}$ (Buriol et al. 2007). Nos meses de inverno, a temperatura mínima média é menor do que $10^{\circ} \mathrm{C}$ (IBGE 1986).

O presente estudo foi realizado em 101 sítios pertencentes a 13 microbacias localizadas em dois trechos da bacia do Rio Ibicuí (Tabela 1, Figura 1). O Trecho 1 corresponde ao curso superior da bacia, localiza-se na Região Central do Estado, e está situado em uma área de encosta, entre o Planalto Riograndense e a Depressão Central (Hundertmarck \& Miorin 2001). Está representado pela sub-bacia do Rio Toropi (6 $6^{\mathrm{a}}$ ordem), com área de drenagem de $47.740 \mathrm{~km}^{2}$ (Paiva et al. 2000), e apresenta substrato constituído de cascalho na encosta, e arenoso à medida 


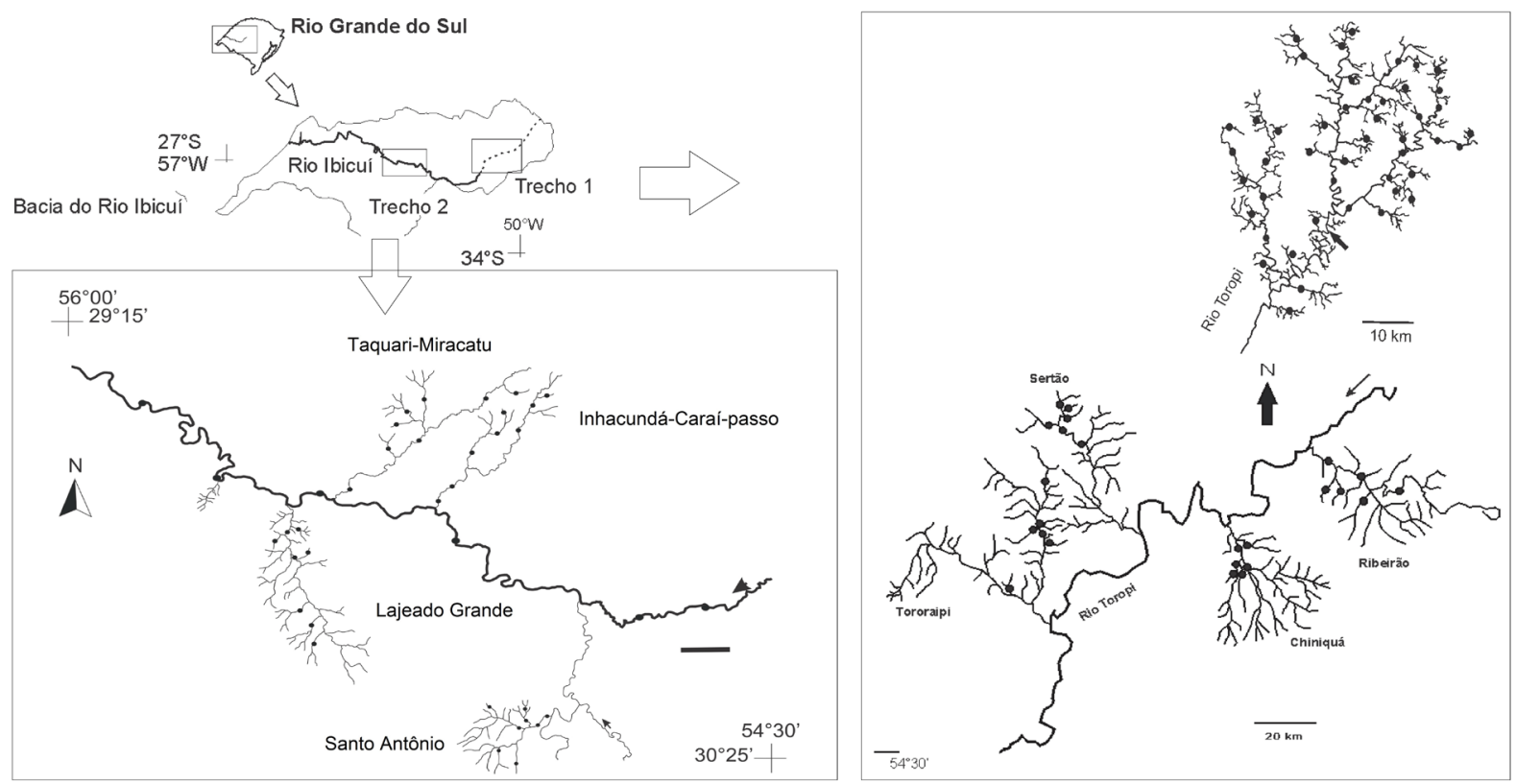

Figura 1. Localização da bacia do Rio Ibicuí, RS, Brasil e dos trechos de coleta. Trecho 1: curso superior (sub-bacia do Rio Toropi); Trecho 2: curso médio-inferior.

que adentra a Depressão (Vieira 1984). A vegetação é constituída pela Floresta Estacional Decidual, típica do Bioma Mata Atlântica (Cordeiro \& Hasenack 2009). A agropecuária é a atividade econômica predominante na região, representada por culturas cíclicas (arroz e soja) e pastagens (IBGE 1986). Os riachos amostrados nesta região localizam-se nos municípios de Mata, São Pedro, Quevedos, Toropi e Tupanciretã 0 Trecho 2 corresponde ao curso médio-inferior da bacia do Rio Ibicuí, localiza-se na Região da Fronteira Oeste do RS, ocupando uma grande área da Depressão Central. Este inclui o leito principal do Rio Ibicuí, de baixa correnteza e substrato tipicamente arenoso (Rambo 1994). A vegetação predominante corresponde à Savana-estépica, típica do Bioma Pampa (Cordeiro \& Hasenack 2009). A agropecuária, especialmente o cultivo de arroz irrigado, é a principal atividade econômica nesta área da bacia (Paiva et al. 2000). . Os riachos amostrados nesta região localizam-se nos municípios de Alegrete, Cacequi, Manoel Viana, Rosário do Sul e São Vicente do Sul.

\subsection{Amostragem e análise de dados}

As amostragens foram realizadas em 101 locais da bacia do Rio Ibicuí, nos anos de 2009 e 2010, durante os meses de primavera e outono, quando os períodos de seca e de cheias são mais raros. No Trecho 1 , foram amostrados 62 locais incluindo o canal principal do Rio Toropi e riachos tributários. No Trecho 2, foram amostrados 39 locais localizados no canal principal do Rio Ibicuí e em riachos tributários. Em todos os locais de coleta os espécimes foram coletados varrendo-se o substrato das margens com auxílio de peneiras de malha $1 \mathrm{~mm}$. Em cada local as amostragens foram feitas em três parcelas de $1 \times 5 \mathrm{~m}$ cada. A vegetação aquática presente no interior de cada parcela vistoriada e os exemplares nelas encontrados foram adicionados às amostras. Em laboratório, a triagem do material foi realizada com auxílio de estereomicroscópio e todos os espécimes foram identificados até gênero, com o auxílio de bibliografia especializada (Costa et al. 2004, Heckman et al. 2008) e, eventualmente, de especialistas. Os espécimes foram preservados em álcool $70 \%$, e estão depositados na Coleção de Invertebrados do Departamento de Biologia, da Universidade Federal de Santa Maria, Rio Grande do Sul, Brasil, sob a seguinte numeração: IA2141 - IA2156; IA2157 - IA2213; IA2214 - IA2266; IA2267 - IA2321; IA2322 - IA2344; IA2345 - IA2358; IA2359 - 2391; IA2392 - IA2480; IA2481 - IA2494; e IA2495 - IA2541.

Riqueza (S), número de indivíduos coletados $(\mathrm{N})$ e frequência de ocorrência $\left(\mathrm{FO}\right.$; em $\%=\mathrm{n}^{\mathrm{o}}$ de pontos de ocorrência/ $\mathrm{n}^{\mathrm{o}}$ total de pontos amostrados) de cada gênero foram analisados para a área total do estudo. Para os dois trechos e para a área total de estudo, a riqueza cumulativa de gêneros foi calculada através da construção de uma curva do coletor, baseada em 500 curvas geradas por adição aleatória das amostras (Colwell \& Coddington 1994). A análise foi realizada utilizando-se o programa EstimateS, versão 8.2 (Colwell 2009).

Curvas de rarefação foram construídas em função do número de indivíduos por parcela, de forma a possibilitar a comparação da riqueza de gêneros entre os trechos estudados, em níveis comparáveis de esforço amostral (Gotelli \& Colwell 2001). As curvas foram 
Tabela 1. Localização dos locais de coleta de larvas de Odonata na bacia do Rio Ibicuí, estado do Rio Grande do Sul, Brasil.

\begin{tabular}{|c|c|c|c|c|c|}
\hline Local & Localização geográfica & Local & Localização geográfica & Local & Localização geográfica \\
\hline Trecho 1 & & Trecho 1 & & Trecho 2 & \\
\hline 1 & $\begin{array}{c}29^{\circ} 36^{\prime} 8 \mathrm{~S} \\
54^{\circ} 28^{\prime} 58 \mathrm{~W}\end{array}$ & 32 & $29^{\circ} 26^{\prime} 420 \mathrm{~S} 53^{\circ} 56^{\prime} 238 \mathrm{~W}$ & 63 & $29^{\circ} 48^{\prime} 23 \mathrm{~S} 54^{\circ} 39^{\prime} 09 \mathrm{~W}$ \\
\hline 2 & $29^{\circ} 36^{\prime} 500 \mathrm{~S} 54^{\circ} 20^{\prime} 523 \mathrm{~W}$ & 33 & $29^{\circ} 18^{\prime} 372 \mathrm{~S} 53^{\circ} 48^{\prime} 574 \mathrm{~W}$ & 64 & $29^{\circ} 50^{\prime} 23 \mathrm{~S} 54^{\circ} 47^{\prime} 55 \mathrm{~W}$ \\
\hline 3 & $29^{\circ} 33^{\prime} 314 \mathrm{~S} 54^{\circ} 29^{\prime} 092 \mathrm{~W}$ & 34 & $29^{\circ} 09^{\prime} 308 \mathrm{~S} \mathrm{53} 44^{\prime} 497 \mathrm{~W}$ & 65 & $29^{\circ} 41^{\prime} 01 \mathrm{~S} 55^{\circ} 11^{\prime} 31 \mathrm{~W}$ \\
\hline 4 & $29^{\circ} 36^{\prime} 423 \mathrm{~S} 54^{\circ} 21^{\prime} 042 \mathrm{~W}$ & 35 & $29^{\circ} 16^{\prime} 257 \mathrm{~S} 53^{\circ} 47^{\prime} 568 \mathrm{~W}$ & 66 & $29^{\circ} 35^{\prime} 52 \mathrm{~S} 55^{\circ} 29^{\prime} 11 \mathrm{~W}$ \\
\hline 5 & $29^{\circ} 35^{\prime} 075 \mathrm{~S} 54^{\circ} 29^{\prime} 202 \mathrm{~W}$ & 36 & $29^{\circ} 05^{\prime} 485 \mathrm{~S} 53^{\circ} 58^{\prime} 293 \mathrm{~W}$ & 67 & $29^{\circ} 33^{\prime} 53 \mathrm{~S} 55^{\circ} 40^{\prime} 42 \mathrm{~W}$ \\
\hline 6 & $29^{\circ} 36^{\prime} 379 \mathrm{~S} 54^{\circ} 20^{\prime} 403 \mathrm{~W}$ & 37 & $29^{\circ} 06^{\prime} 445 \mathrm{~S} 53^{\circ} 50^{\prime} 062 \mathrm{~W}$ & 68 & $29^{\circ} 24^{\prime} 56 \mathrm{~S} 55^{\circ} 51^{\prime} 02 \mathrm{~W}$ \\
\hline 7 & $29^{\circ} 38^{\prime} 354 \mathrm{~S} 54^{\circ} 29^{\prime} 064 \mathrm{~W}$ & 38 & $29^{\circ} 03^{\prime} 586 \mathrm{~S} 53^{\circ} 56^{\prime} 261 \mathrm{~W}$ & 69 & $30^{\circ} 15^{\prime} 58 \mathrm{~S} 55^{\circ} 01^{\prime} 10 \mathrm{~W}$ \\
\hline 8 & $29^{\circ} 43^{\prime} 005 \mathrm{~S} 54^{\circ} 30^{\prime} 285 \mathrm{~W}$ & 39 & $29^{\circ} 24^{\prime} 270 \mathrm{~S} 53^{\circ} 42^{\prime} 573 \mathrm{~W}$ & 70 & $30^{\circ} 19^{\prime} 51 \mathrm{~S} 54^{\circ} 59^{\prime} 39 \mathrm{~W}$ \\
\hline 9 & $29^{\circ} 33^{\prime} 311 \mathrm{~S} 54^{\circ} 29^{\prime} 098 \mathrm{~W}$ & 40 & $29^{\circ} 09^{\prime} 095 \mathrm{~S} 53^{\circ} 58^{\prime} 058 \mathrm{~W}$ & 71 & $30^{\circ} 16^{\prime} 07 \mathrm{~S} 54^{\circ} 58^{\prime} 45 \mathrm{~W}$ \\
\hline 10 & $29^{\circ} 31^{\prime} 309 \mathrm{~S} 54^{\circ} 13^{\prime} 566 \mathrm{~W}$ & 41 & $\begin{array}{c}29^{\circ} 35^{\prime} 36 \mathrm{~S} \\
54^{\circ} 29^{\prime} 26 \mathrm{~W}\end{array}$ & 72 & $30^{\circ} 19^{\prime} 31 \mathrm{~S} 55^{\circ} 01^{\prime} 04 \mathrm{~W}$ \\
\hline 11 & $29^{\circ} 26^{\prime} 317 \mathrm{~S} 54^{\circ} 12^{\prime} 488 \mathrm{~W}$ & 42 & $\begin{array}{l}29^{\circ} 35^{\prime} 36 \mathrm{~S} \\
54^{\circ} 29^{\prime} 28 \mathrm{~W}\end{array}$ & 73 & $30^{\circ} 17^{\prime} 42 \mathrm{~S} 54^{\circ} 59^{\prime} 17 \mathrm{~W}$ \\
\hline 12 & $29^{\circ} 27^{\prime} 130 \mathrm{~S} 54^{\circ} 09^{\prime} 551 \mathrm{~W}$ & 43 & $\begin{array}{l}29^{\circ} 37^{\prime} 40 \mathrm{~S} \\
54^{\circ} 30^{\prime} 60 \mathrm{~W}\end{array}$ & 74 & $30^{\circ} 18^{\prime} 46 \mathrm{~S} 54^{\circ} 59^{\prime} 49 \mathrm{~W}$ \\
\hline 13 & $29^{\circ} 21^{\prime} 004 \mathrm{~S} 54^{\circ} 06^{\prime} 090 \mathrm{~W}$ & 44 & $29^{\circ} 34^{\prime} 227 \mathrm{~S} 54^{\circ} 15^{\prime} 922 \mathrm{~W}$ & 75 & $30^{\circ} 17^{\prime} 01 \mathrm{~S} 54^{\circ} 57^{\prime} 43 \mathrm{~W}$ \\
\hline 14 & $29^{\circ} 28^{\prime} 397 \mathrm{~S} 54^{\circ} 14^{\prime} 009 \mathrm{~W}$ & 45 & $29^{\circ} 33^{\prime} 796 \mathrm{~S} 54^{\circ} 16^{\prime} 83 \mathrm{~W}$ & 76 & $30^{\circ} 16^{\prime} 46 \mathrm{~S} 54^{\circ} 57^{\prime} 31 \mathrm{~W}$ \\
\hline 15 & $29^{\circ} 27^{\prime} 402 \mathrm{~S} 54^{\circ} 11^{\prime} 379 \mathrm{~W}$ & 46 & $29^{\circ} 32^{\prime} 731 \mathrm{~S} 54^{\circ} 17^{\prime} 497 \mathrm{~W}$ & 77 & $29^{\circ} 40^{\prime} 43 \mathrm{~S} 55^{\circ} 33^{\prime} 03 \mathrm{~W}$ \\
\hline 16 & $29^{\circ} 30^{\prime} 565 \mathrm{~S} 54^{\circ} 09^{\prime} 293 \mathrm{~W}$ & 47 & $29^{\circ} 33^{\prime} 792 \mathrm{~S} 54^{\circ} 17^{\prime} 407 \mathrm{~W}$ & 78 & $29^{\circ} 43^{\prime} 30 \mathrm{~S} 55^{\circ} 30^{\prime} 57 \mathrm{~W}$ \\
\hline 17 & $29^{\circ} 31^{\prime} 185 \mathrm{~S} 54^{\circ} 06^{\prime} 022 \mathrm{~W}$ & 48 & $29^{\circ} 33^{\prime} 281 \mathrm{~S} 54^{\circ} 15^{\prime} 996 \mathrm{~W}$ & 79 & $29^{\circ} 39^{\prime} 54 \mathrm{~S} 55^{\circ} 31^{\prime} 53 \mathrm{~W}$ \\
\hline 18 & $29^{\circ} 32^{\prime} 340 \mathrm{~S} 54^{\circ} 08^{\prime} 154 \mathrm{~W}$ & 49 & $29^{\circ} 33^{\prime} 792 \mathrm{~S} 54^{\circ} 14^{\prime} 449 \mathrm{~W}$ & 80 & $29^{\circ} 54^{\prime} 55 \mathrm{~S} 55^{\circ} 29^{\prime} 31 \mathrm{~W}$ \\
\hline 19 & $29^{\circ} 25^{\prime} 068 \mathrm{~S} 54^{\circ} 05^{\prime} 294 \mathrm{~W}$ & 50 & $29^{\circ} 36^{\prime} 733 \mathrm{~S} 54^{\circ} 21^{\prime} 09 \mathrm{~W}$ & 81 & $29^{\circ} 49^{\prime} 34 \mathrm{~S} 55^{\circ} 30^{\prime} 44 \mathrm{~W}$ \\
\hline 20 & $29^{\circ} 32^{\prime} 538 \mathrm{~S} 54^{\circ} 09^{\prime} 062 \mathrm{~W}$ & 51 & $29^{\circ} 35^{\prime} 903 \mathrm{~S} 54^{\circ} 20^{\prime} 69 \mathrm{~W}$ & 82 & $29^{\circ} 51^{\prime} 16 \mathrm{~S} 55^{\circ} 29^{\prime} 19 \mathrm{~W}$ \\
\hline 21 & $29^{\circ} 21^{\prime} 008 \mathrm{~S} 54^{\circ} 12^{\prime} 377 \mathrm{~W}$ & 52 & $29^{\circ} 35^{\prime} 993 \mathrm{~S} 54^{\circ} 20^{\prime} 964 \mathrm{~W}$ & 83 & $29^{\circ} 43^{\prime} 18 \mathrm{~S} 55^{\circ} 32^{\prime} 03 \mathrm{~W}$ \\
\hline 22 & $29^{\circ} 26^{\prime} 545 \mathrm{~S} 54^{\circ} 07^{\prime} 463 \mathrm{~W}$ & 53 & $29^{\circ} 31^{\prime} 630 \mathrm{~S} 54^{\circ} 29^{\prime} 159 \mathrm{~W}$ & 84 & $29^{\circ} 40^{\prime} 42 \mathrm{~S} 55^{\circ} 32^{\prime} 58 \mathrm{~W}$ \\
\hline 23 & $29^{\circ} 19^{\prime} 062 \mathrm{~S} 54^{\circ} 09^{\prime} 157 \mathrm{~W}$ & 54 & $29^{\circ} 31^{\prime} 747 \mathrm{~S} 54^{\circ} 28^{\prime} 467 \mathrm{~W}$ & 85 & $29^{\circ} 23^{\prime} 08 \mathrm{~S} 55^{\circ} 00^{\prime} 55 \mathrm{~W}$ \\
\hline 24 & $29^{\circ} 28^{\prime} 593 \mathrm{~S} 54^{\circ} 04^{\prime} 181 \mathrm{~W}$ & 55 & $\begin{array}{c}29^{\circ} 32^{\prime} 183 \\
54^{\circ} 27^{\prime} 581 \mathrm{~W}\end{array}$ & 86 & $29^{\circ} 25^{\prime} 54 \mathrm{~S} 55^{\circ} 07^{\prime} 41 \mathrm{~W}$ \\
\hline 25 & $29^{\circ} 16^{\prime} 572 \mathrm{~S} 54^{\circ} 05^{\prime} 577 \mathrm{~W}$ & 56 & $29^{\circ} 31^{\prime} 182 \mathrm{~S} 54^{\circ} 28^{\prime} 284 \mathrm{~W}$ & 87 & $29^{\circ} 25^{\prime} 23 \mathrm{~S} 55^{\circ} 03^{\prime} 21 \mathrm{~W}$ \\
\hline 26 & $29^{\circ} 19^{\prime} 359 \mathrm{~S} 54^{\circ} 03^{\prime} 286 \mathrm{~W}$ & 57 & $29^{\circ} 30^{\prime} 568 \mathrm{~S} 54^{\circ} 28^{\prime} 572 \mathrm{~W}$ & 88 & $29^{\circ} 27^{\prime} 48 \mathrm{~S} 55^{\circ} 07^{\prime} 14 \mathrm{~W}$ \\
\hline 27 & $29^{\circ} 19^{\prime} 049 \mathrm{~S} 54^{\circ} 07^{\prime} 200 \mathrm{~W}$ & 58 & $29^{\circ} 30^{\prime} 662 \mathrm{~S} 54^{\circ} 28^{\prime} 514 \mathrm{~W}$ & 89 & $29^{\circ} 25^{\prime} 79 \mathrm{~S} 55^{\circ} 04^{\prime} 12 \mathrm{~W}$ \\
\hline 28 & $29^{\circ} 22^{\prime} 565 \mathrm{~S} 54^{\circ} 03^{\prime} 466 \mathrm{~W}$ & 59 & $29^{\circ} 39^{\prime} 955 \mathrm{~S} 54^{\circ} 28^{\prime} 291 \mathrm{~W}$ & 90 & $29^{\circ} 31^{\prime} 02 \mathrm{~S} 55^{\circ} 10^{\prime} 43 \mathrm{~W}$ \\
\hline 29 & $29^{\circ} 14^{\prime} 349 \mathrm{~S} 53^{\circ} 57^{\prime} 586 \mathrm{~W}$ & 60 & $29^{\circ} 38^{\prime} 249$ S $54^{\circ} 28^{\prime} 665 \mathrm{~W}$ & 91 & $29^{\circ} 35^{\prime} 28 \mathrm{~S} 55^{\circ} 14^{\prime} 01 \mathrm{~W}$ \\
\hline 30 & $29^{\circ} 11^{\prime} 272 \mathrm{~S} 54^{\circ} 05^{\prime} 067 \mathrm{~W}$ & 61 & $29^{\circ} 38^{\prime} 247 \mathrm{~S} 54^{\circ} 28^{\prime} 803 \mathrm{~W}$ & 92 & $29^{\circ} 36^{\prime} 22 \mathrm{~S} 55^{\circ} 14^{\prime} 10 \mathrm{~W}$ \\
\hline \multirow[t]{9}{*}{31} & $29^{\circ} 06^{\prime} 194 \mathrm{~S} 53^{\circ} 56^{\prime} 391 \mathrm{~W}$ & 62 & $29^{\circ} 43^{\prime} 847 \mathrm{~S} 54^{\circ} 20^{\prime} 964 \mathrm{~W}$ & 93 & $29^{\circ} 33^{\prime} 35 \mathrm{~S} 55^{\circ} 04^{\prime} 33 \mathrm{~W}$ \\
\hline & & & & 94 & $29^{\circ} 26^{\prime} 25 \mathrm{~S} 55^{\circ} 18^{\prime} 30 \mathrm{~W}$ \\
\hline & & & & 95 & $29^{\circ} 28^{\prime} 30 \mathrm{~S} 55^{\circ} 18^{\prime} 05 \mathrm{~W}$ \\
\hline & & & & 96 & $29^{\circ} 23^{\prime} 33 \mathrm{~S} 55^{\circ} 08^{\prime} 28 \mathrm{~W}$ \\
\hline & & & & 97 & $29^{\circ} 25^{\prime} 06 \mathrm{~S} 55^{\circ} 18^{\prime} 47 \mathrm{~W}$ \\
\hline & & & & 98 & $29^{\circ} 24^{\prime} 36 \mathrm{~S} 55^{\circ} 16^{\prime} 01 \mathrm{~W}$ \\
\hline & & & & 99 & $29^{\circ} 28^{\prime} 42 \mathrm{~S} 55^{\circ} 16^{\prime} 39 \mathrm{~W}$ \\
\hline & & & & 100 & $29^{\circ} 35^{\prime} 13 \mathrm{~S} 55^{\circ} 25^{\prime} 23 \mathrm{~W}$ \\
\hline & & & & 101 & $29^{\circ} 33^{\prime} 59 \mathrm{~S} 54^{\circ} 41^{\prime} 31 \mathrm{~W}$ \\
\hline
\end{tabular}


Tabela 2. Composição, riqueza, número de indivíduos coletados $(\mathrm{N})$ e frequência de ocorrência $(\mathrm{FO})$ dos gêneros de larva de Odonata amostrados na área total de estudo e nos trechos 1 e 2, na bacia do Rio Ibicuí.

\begin{tabular}{|c|c|c|c|c|c|c|}
\hline \multirow[t]{2}{*}{ Famílias/Gêneros } & \multicolumn{2}{|c|}{ Trecho 1} & \multicolumn{2}{|c|}{ Trecho 2} & \multicolumn{2}{|c|}{ Área total de estudo } \\
\hline & $\mathrm{N}$ & FO (\%) & $\mathrm{N}$ & FO (\%) & $\mathrm{N}$ & FO $(\%)$ \\
\hline \multicolumn{7}{|l|}{ Aeshnidae } \\
\hline Coryphaeschna Williamson, 1903 & 3 & 3,12 & 0 & & 3 & 1,8 \\
\hline Rhionaeschna Förster, 1909 & 1 & 1,56 & 2 & 2,63 & 3 & 1,8 \\
\hline \multicolumn{7}{|l|}{ Corduliidae } \\
\hline Navicordulia Machado e Costa, 1995 & 13 & 14,06 & 46 & 28,94 & 59 & 17,9 \\
\hline \multicolumn{7}{|l|}{ Gomphidae } \\
\hline Agriogomphus Selys, 1869 & 0 & & 1 & 2,63 & 1 & 0,9 \\
\hline Aphylla Selys, 1854 & 8 & 6,25 & 4 & 5,26 & 12 & 5,6 \\
\hline Archaeogomphus Williamson, 1919 & 0 & & 2 & 5,26 & 2 & 1,8 \\
\hline Cacoides Cowley, 1934 & 12 & 6,25 & 1 & 2,63 & 13 & 4,7 \\
\hline Desmogomphus Williamson, 1920 & 24 & 12,5 & 11 & 13,15 & 35 & 9,4 \\
\hline Phyllocycla Calvert, 1948 & 47 & 10,93 & 30 & 28,94 & 77 & 17,9 \\
\hline Progomphus Selys, 1854 & 196 & 35,93 & 68 & 36,31 & 264 & 28,3 \\
\hline Tibiogomphus Belle, 1992 & 4 & 4,68 & 2 & 2,63 & 6 & 3,7 \\
\hline \multicolumn{7}{|l|}{ Libellulidae } \\
\hline Brechmorhoga Kirby 1894 & 6 & 6,25 & 7 & 1,67 & 13 & 8,4 \\
\hline Dasythemis Karsch 1889 & 1 & 1,56 & 14 & 10,52 & 15 & 4,7 \\
\hline Dythemis Hagen, 1861 & 1 & 1,56 & 0 & & 1 & 0,9 \\
\hline Elasmothemis Westfall, 1988 & 4 & 1,56 & 11 & 10,52 & 15 & 3,7 \\
\hline Erythemis Hagen, 1861 & 0 & & 1 & 2,63 & 1 & 0,9 \\
\hline Erythrodiplax Brauer, 1868 & 6 & 7,81 & 52 & 15,78 & 58 & 10,3 \\
\hline Gynothemis Calvert in Ris, 1909 & 0 & & 1 & 2,63 & 1 & 0,9 \\
\hline Idiataphe Cowley, 1934 & 0 & & 1 & 2,63 & 1 & 0,9 \\
\hline Micrathyria Kirby, 1889 & 8 & 4,68 & 9 & 13,15 & 17 & 7,5 \\
\hline Oligoclada Karsch, 1890 & 2 & 3,12 & 0 & & 2 & 1,8 \\
\hline Orthemis Hagen, 1861 & 7 & 4,68 & 6 & 2,63 & 13 & 3,7 \\
\hline Perithemis Hagen, 1861 & 7 & 4,68 & 7 & 7,89 & 14 & 5,6 \\
\hline Tauriphila Kirby, 1889 & 7 & 7,81 & 14 & 13,15 & 21 & 9,4 \\
\hline Tramea Hagen, 1861 & 1 & 1,56 & 4 & 5,26 & 5 & 2,8 \\
\hline \multicolumn{7}{|l|}{ Calopterygidae } \\
\hline Hetaerina Hagen, 1853 & 42 & 25 & 40 & 31,57 & 82 & 23,5 \\
\hline Mnesarete Cowley, 1934 & 2 & 3,12 & 5 & 12,52 & 7 & 7,6 \\
\hline \multicolumn{7}{|l|}{ Coenagrionidae } \\
\hline Acanthagrion Selys, 1876 & 13 & 9,37 & 9 & 7,89 & 22 & 8,4 \\
\hline Argia Rambur, 1842 & 44 & 31,25 & 54 & 15,78 & 98 & 18,8 \\
\hline Homeoura Kennedy, 1920 & 18 & 12,5 & 3 & 7,89 & 21 & 9,4 \\
\hline Ischnura Charpentier, 1840 & 2 & 3,12 & 0 & & 2 & 1,8 \\
\hline Oxyagrion Selys, 1876 & 8 & 3,12 & 6 & 7,89 & 14 & 5,6 \\
\hline Telebasis Selys, 1865 & 2 & 3,12 & 0 & & 2 & 2,8 \\
\hline \multicolumn{7}{|l|}{ Megapodagrionidae } \\
\hline Heteragrion Selys, 1862 & 1 & 1,56 & 0 & & 1 & 0,9 \\
\hline Total & & 546 & & 418 & & 964 \\
\hline Riqueza & & 31 & & 28 & & 34 \\
\hline
\end{tabular}


geradas utilizando-se o programa EcoSim, versão 7.72 (Colwell et al. 2004). É importante ressaltar que esta abordagem analítica produz estimativas de riqueza de espécies baseadas nas amostras, e, portanto, por unidade de área, tanto quanto estimativas baseadas no número de indivíduos amostrados.

\section{Resultados}

Ao todo, 964 indivíduos foram encontrados na área inventariada, pertencentes a 34 gêneros e distribuídos em sete famílias (Tabela 2). A subordem Anisoptera foi representada por quatro famílias (Aeshnidae, Corduliidae, Gomphidae e Libellulidae), enquanto a subordem Zygoptera, por três (Calopterygidae, Coenagrionidae e Megapodagrionidae). A família mais diversa foi Libellulidae, representada por 14 gêneros, e a mais abundante, Gomphidae, com
410 indivíduos. Progomphus Selys, 1854 (Gomphidae) foi o gênero mais abundante, com 264 indivíduos. Dentre os zigópteros, a família mais representativa foi Coenagrionidae, com seis gêneros, sendo Argia Rambur, 1842, o mais abundante, com 98 indivíduos. Os gêneros com maior frequência de ocorrência na área total de estudo foram Progomphus (28,3\%), Hetaerina Hagen in Selys, 1853 (23,5\%) e Argia (18,8\%). Os gêneros Agriogomphus Selys, 1869, Archaeogomphus Williamson, 1919, Dythemis Hagen, 1861, Erythemis Hagen, 1861, Gynothemis Calvert, 1909, Idiataphe Cowley, 1934, Oligoclada Karsch, 1890, Ischnura Charpentier, 1840, Telebasis Selys, 1865 e Heteragrion Selys, 1862 foram considerados raros por terem sido representados por menos de três indivíduos em toda área de estudo.

O Trecho 1 (encosta) apresentou maior abundância (546 indivíduos) e maior riqueza (31 gêneros) em relação ao Trecho 2 (planície). No Trecho 1, o gênero
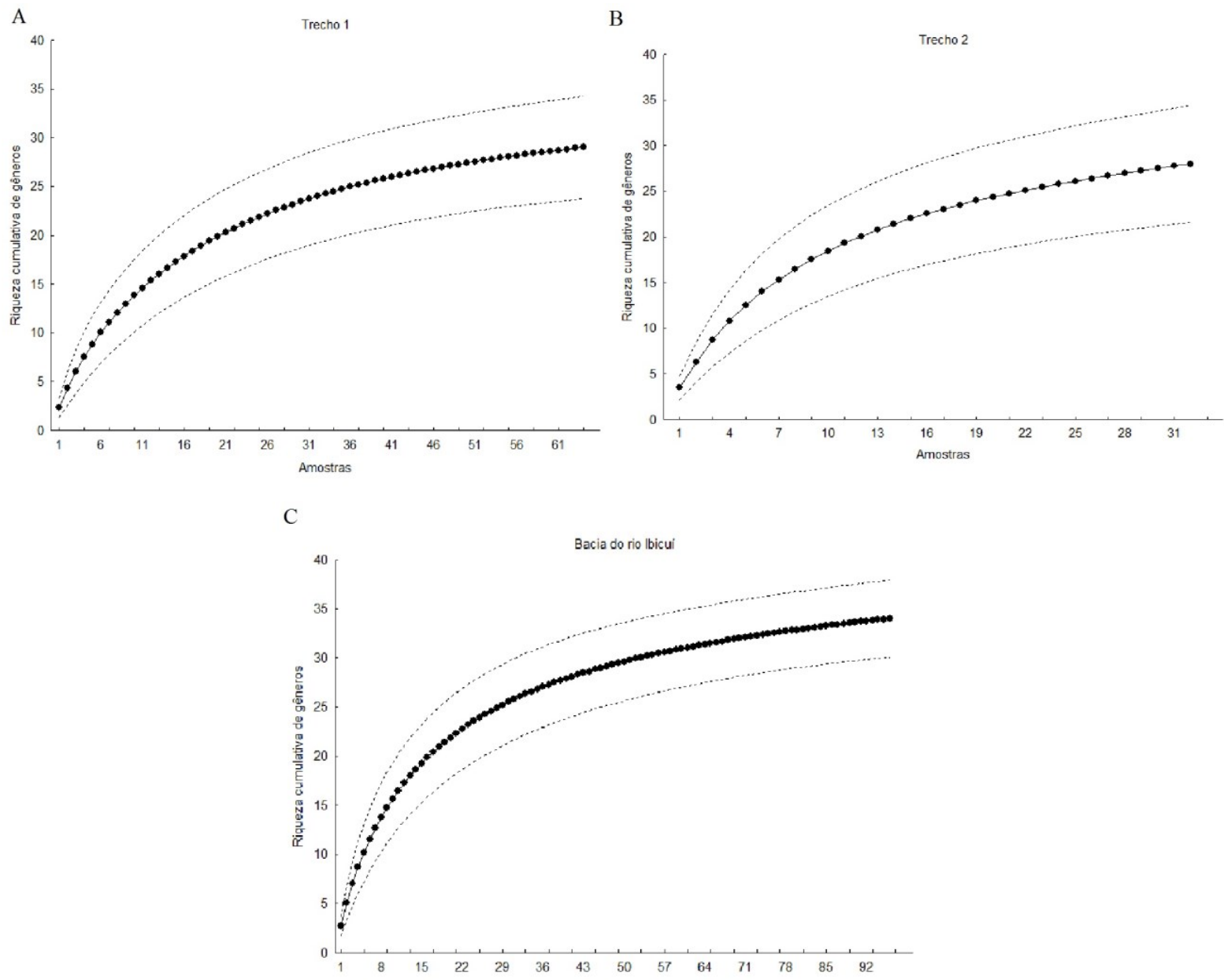

Figura 2. Curvas de acumulação de gêneros de larvas de Odonata coletados nos trechos 1 (a) e 2 (b), na bacia do rio Ibicuí (c), RS, Brasil. As linhas com pontos escuros representam o valor médio calculado e as linhas pontilhadas indicam os seus respectivos intervalos de confiança (95\%). 


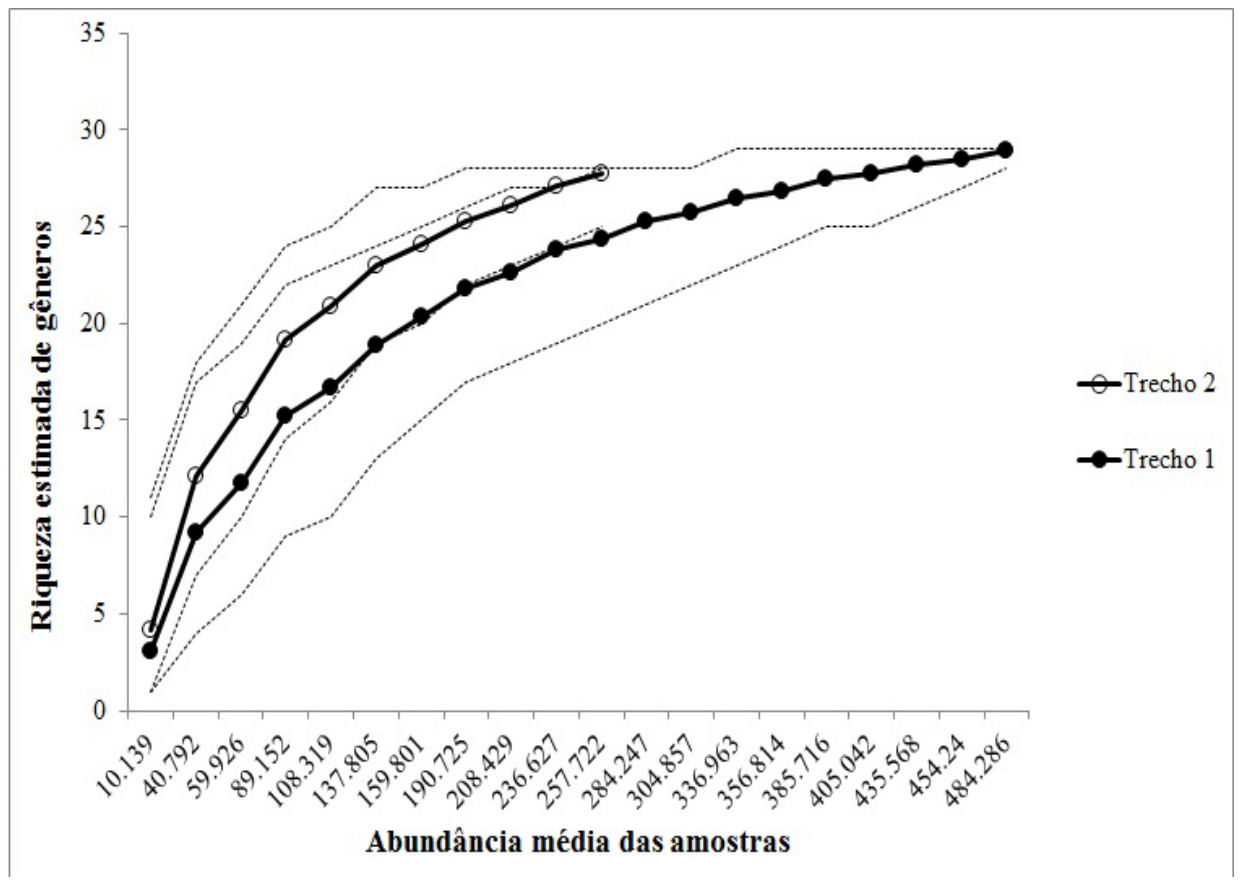

Figura 3. Comparação da riqueza estimada de gêneros de larvas de Odonata entre os trechos de estudo na bacia do Rio Ibicuí, RS, Brasil.

mais abundante foi Progomphus (196 indivíduos) e a família mais diversa, Libellulidae (11 gêneros). Os gêneros Coryphaeschna, Dythemis, Heteragrion, Ischnura, Oligoclada, Telebasis foram exclusivos deste trecho. No Trecho 2, o gênero mais abundante também foi Progomphus (68 indivíduos), e a família mais rica, também foi Libellulidae (12 gêneros). Os gêneros Agriogomphus, Archaegomphus, Erythemis, Gynothemis e Idiataphe foram exclusivos dos riachos deste trecho.

As curvas de acumulação de gêneros para cada trecho do estudo, bem como para a área como um todo, não atingiram a assíntota, apesar de apresentarem uma tendência à estabilização (Figuras 2a, $2 b$ e 2c). Não foi observada diferença significativa na riqueza média de gêneros estimada entre os trechos estudados (Figura 3).

\section{Discussão}

Dos 34 gêneros encontrados neste trabalho, Agriogomphus, Cacoides (Gomphidae) e Mnesarete (Calopterygidae) são novos registros para o Rio Grande do Sul. Adicionando-se o registro destes novos gêneros aos inventários previamente realizados no Estado (Costa et al. 1971, Teixeira et al. 1971, Maltchik et al. 2010), a diversidade total de Odonata conhecida no Rio Grande do Sul passa a compreender 40 gêneros. A distribuição de Agriogomphus e Cacoides foi estendida para regiões subtropicais, uma vez que eram conhecidos somente para regiões tropicais dos neotrópicos (América Central e norte dos Andes, no primeiro caso, e Amazônia e Nordeste brasileiro, no segundo) (Garrison et al. 2006).

A riqueza total de gêneros registrada para o Estado é menor do que a de estados da Região Sudeste do país. Estudos prévios mostram que em estados como São Paulo, Espírito Santo e Rio de Janeiro ocorrem 78 (Costa et al. 2000), 69 (Costa \& Oldrini 2005) e 62 (Carvalho e Nessimian 1998) gêneros de odonatas, respectivamente. A menor riqueza geral registrada no Rio Grande do Sul deve estar associada à predominância de temperaturas mais frias na região Sul (média mínima $<10{ }^{\circ} \mathrm{C}$ no inverno; IBGE, 1986). Uma vez que um maior número de espécies de Odonata é registrado em áreas tropicais, tal padrão reflete um gradiente de riqueza para a ordem relacionado à temperatura (Kalkman et al. 2008). Ao mesmo tempo, a baixa riqueza registrada no RS pode ser decorrência dos poucos estudos (subamostragem) feitos no Estado (De Marco \& Vianna 2005), de maneira similar ao que ocorre em estados com clima tropical pouco amostrados (23 gêneros no Estado do Mato Grosso do Sul, oito gêneros no Estado do Mato Grosso e 34 gêneros no Estado de Roraima; Machado et al. 1991, Juen et al. 2007, Dalzochio et al. 2011). 
Contudo, ao considerar apenas trabalhos realizados em ambientes lóticos, verifica-se que a riqueza de gêneros de Odonata é maior na bacia Rio Ibicuí do que em riachos de bacias localizadas em regiões tropicais e subtropicais do país, como a Sudeste e Centro-Oeste (14, Ferreira-Peruquetti \& De Marco 2002; 22, Ferreira-Peruquetti \& Gessner 2003 e 14, Assis et al. 2004; 30, Juen et al 2007, 14, Dalzochio et al. 2011). A maior riqueza registrada na área aqui estudada pode estar associada ao grande esforço amostral despendido às amostragens e à heterogeneidade ambiental da área de estudo. Porém, se considerarmos o total de gêneros encontrados nos outros estudos por região, a riqueza encontrada na bacia do Rio Ibicuí continua sendo maior. Assim, o resultado obtido no presente estudo corrobora a tendência de ambientes lóticos de regiões com clima temperado possuírem riqueza de certas comunidades de macroinvertebrados maior do que as de regiões com clima mais quente (McKie et al. 2005, Floss et al. 2012). Uma alta riqueza de odonatas (mais de 230 espécies) é registrada para a Argentina (Rodríguez-Capítulo 1992), cujo território é amplamente situado em áreas temperadas. Porém, não se pode descartar que a localização da bacia do Rio Ibicuí, inserida em dois biomas (Pampa e Mata Atlântica), propicie uma maior diversidade de microhabitats e, consequentemente, maior riqueza de gêneros. A maior parte dos estudos sobre odonatas conduzidos no Brasil está restrita a um único bioma ou a um trecho específico de uma determinada bacia. Portanto, estes podem ter sido feitos em áreas com menor heterogeneidade de habitats, o que pode ter determinado os menores valores de riqueza neles registrados.

Sete das 21 famílias de odonatas conhecidas para a região Neotropical foram registradas neste trabalho. De modo geral, Libellulidae é a família mais rica nos neotrópicos, com 38 gêneros (Kalkman et al. 2008). Neste trabalho a família de maior riqueza também foi Libellulidae (41,17\% do total). A predominância desta família também foi descrita em riachos de outros estados do Brasil (Ferreira-Peruquetti \& De Marco 2002, Ferreira-Peruquetti \& Gessner 2003, Assis et al. 2004, Dalzochio et al. 2011).

Progomphus (28,3\%), Hetaerina (22,5\%) e Argia (18,8\%) foram os gêneros com maior frequência de ocorrência na área total de estudo. Hetaerina e Argia também predominam em riachos de outras regiões brasileiras (Assis et al. 2004; Dalzochio et al. 2011). A dominância destes dois gêneros na bacia do Rio Ibicuí pode ser explicada por serem exclusivos de ambientes lóticos e, adicionalmente, pelos hábitos de seus estágios larvais. As larvas das espécies de Argia, além de serem típicas de áreas montanhosas (Dalzochio et al. 2011), possuem hábito reptante (Carvalho \& Nessimian
1998) que permite a ocupação de variados tipos de substrato, como os encontrados na bacia aqui estudada (pedregoso na área de encosta; arenoso na planície). As larvas das espécies de Hetaerina são descritas como agarradoras (Carvalho \& Nessimian 1998), e associadas tanto com os detritos de áreas de encosta, quanto com o material carregado e depositado nas áreas de planície. Além disso, são geralmente registradas em todos os trechos de uma bacia, e em áreas com condições ambientais heterogêneas (Ferreira-Peruquetti \& De Marco 2002, Costa et al. 2004). A predominância de Progomphus também foi registrada em riachos (Kikuchi \& Uieda 2005) de outras regiões do Brasil, em ambientes com substratos arenosos e cascalhosos (Assis et al. 2004). A alta frequência deste gênero na área total aqui estudada, especificamente no Trecho 2, pode ser explicada pelo hábito fossorial de suas espécies (Carvalho \& Nessimian 1998), que favorece a ocorrência em riachos com substrato sedimentar (Assis et al. 2004), tais como os da bacia do Rio Ibicuí.

A assíntota da curva do coletor não foi atingida em nenhum dos trechos, nem para a área como um todo. A ocorrência de muitos gêneros raros $(29,5 \%)$ pode ter contribuído para este resultado. Adicionalmente, áreas de remanso marginais não foram amostradas, o que pode ter contribuído para a ausência e/ ou menores frequências de alguns gêneros típicos deste microhabitat, especialmente aqueles associados à macrófitas. Em termos de composição, algumas diferenças podem ser destacadas com relação ao estudo conduzido em áreas úmidas de todo o RS (Maltchik et al. 2010), no qual 28 gêneros de odonatas, pertencentes a cinco famílias, foram encontrados. As famílias mais ricas foram Libellulidae, Coenagrionidae e Aeshnidae, e os gêneros mais abundantes e amplamente distribuídos, Erythrodiplax Brauer, 1861, Lestes Leach, 1815, Micrathyria Kirby, 1889 e Orthemis Hagen, 1861. Estas famílias e gêneros são comumente registrados na literatura como dominantes em ambientes lênticos e bastante vegetados (De Marco et al. 1999), condições ausentes na área aqui estudada.

Não foram detectadas diferenças nas riquezas médias entre os trechos de encosta (Trecho 1) e planície (Trecho 2) da bacia do Rio Ibicuí. A riqueza total de cada trecho não é muito díspar, mesmo com maior esforço amostral dedicado ao Trecho 1. De um trecho para outro, não ocorreu mudança significativa na composição dos gêneros (cada trecho conteve baixo número de gêneros exclusivos), embora tenha havido modificação entre as frequências de ocorrências de alguns deles, especialmente em Gomphidae (Tabela 2). Diferenças geomorfológicas entre os trechos estudados modulam fatores ambientais de escala local que estão relacionados ao estabelecimento de alguns gêneros no ambiente, como, por exemplo, a preferên- 
cia por determinado tipo de substrato. Este fator, que geralmente varia de montante para jusante (encosta $\mathrm{x}$ planície), pode explicar as modificações observada nas frequências de alguns gêneros, como os de Gomphidae (Carvalho \& Nessimian 1998), na Bacia do Rio Ibicuí. Devido ao hábito fossorial, as larvas desta família estiveram mais bem representadas nos riachos de planície, com substrato arenoso, do que nos riachos da área de encosta, com substrato mais pedregoso.

\section{Agradecimentos}

À MSc. Marina Schmidt Dalzochio (Unisinos), pela ajuda na identificação dos gêneros. À equipe do Laboratório de Macroinvertebrados Aquáticos da UFSM pela colaboração na coleta e triagem do material e fornecimento dos dados abióticos.

\section{Referências}

ASSIS, J. C. F., CARVALHO, A. L. \& NESSIMIAN, J. L. Composição e preferência por microhábitat de imaturos de odonata (Insecta) em um trecho de baixada do Rio Ubatiba, Maricá-RJ, Brasil. Revista Brasileira de Entomologia, Curitiba, v. 48, p.273282, 2004.

BURIOL, G. A. et al. Clima e vegetação natural do estado do Rio Grande do Sul segundo o diagrama climático de Walter e Lieth. Ciência Florestal, Santa Maria, v. 17, n. 2, p. 91-100, 2007.

CARVALHO, A. L. \& NESSIMIAN, J. L. 1998. Odonata do Estado do Rio de Janeiro, Brasil: Habitats e hábitos das larvas. J.L. Nessimian \& A.L. Carvalho (Eds), Ecologia de Insetos Aquáticos. PPGE-UFRJ. Rio de Janeiro, RJ, Brasil, 1998. P. 3-28.

CLAUSNITZER, V. et al. Odonata enter the biodiversity crisis debate: the first global assessment of an insect group. Biological Conservation, Barking v. 142, p. 1864-1869, 2009.

COLWELL, R. K. EstimateS 8.2.0 for Windows. Connecticut. University of Connecticut, 2009.

COLWELL, R. K., MAO, C. X. \& CHANG, J. Interpolating, extrapolating, and comparing incidence-based species accumulation curves. Ecology, Ithaca, v. 85, p. 2717-2727, 2004.
COLWELL, R. K. \& CODDINGTON, J. A. Estimating terrestrial biodiversity through extrapolation. Philosophical Transactions of the Royal Society of London B, Londres, v. 345, p. 101118, 1994.

CORBET, P. S. Behaviour and Ecology of Odonata. London. Comstock Pub Association, 1999.

CORDEIRO, J. L. P. \& HASENACK, H. Cobertura vegetal atual do Rio Grande do Sul. Pillar, V.P. (ORG.), Campos Sulinos - Conservação e uso sustentável da biodiversidade. Ministério do Meio Ambiente, Brasília, DF, Brasil. 2009. P.285-299.

COSTA, J. M. Contribuição ao conhecimento da fauna odonatológica do município de Santa Maria, Rio Grande do Sul. Atas da Sociedade Biológica do Rio de Janeiro, Rio de Janeiro, v. 14, p. 193-194, 1971.

COSTA, J. M. et al. Diversidade e distribuição dos Odonata (Insecta) no Estado de São Paulo, Brasil: Parte I. Lista preliminar das espécies e registros bibliográficos. Publicações Avulsas do Museu Nacional, Rio de Janeiro, v. 80, p. 1-27, 2000.

COSTA, J. M. \& OLDRINI, B. B. Diversidade e distribuição dos Odonata (Insecta) no estado do Espírito Santo, Brasil. Publicações Avulsas do Museu Nacional, Rio de Janeiro, v. 107, p. 3-15, 2005.

COSTA, J. M., SOUZA, L. O. I. \& OLDRINI, B. B. Chave para Identificação das Famílias e Gêneros das larvas de Odonata do Brasil: Comentários e Registros Bibliográficos (Insecta: Odonata). Publicações Avulsas do Museu Nacional, Rio de Janeiro, v. 99, p. 1-44, 2004.

DALZOCHIO, M. S., COSTA, J. M. \& UCHOAFERNANDES, M. A. Diversity of Odonata (Insecta) in lotic systems from Serra da Bodoquena, Mato Grosso do Sul State, Brazil. Revista Brasileira de Entomologia, Curitiba, v. 55, p. 1-6, 2011.

DE MARCO, P., LATINI, A. O. \& REIS, A. P. Environmental determination of dragonfly assemblage in aquaculture ponds. Aquaculture Research, Oxford, v. 30, p. 357-364, 1999.

DE MARCO, P. \& VIANNA, D. M. Distribuição do esforço de coleta de Odonata no Brasil: subsídios para escolha de áreas prioritárias 
para levantamentos faunísticos. Lundiana, Belo Horizonte, v. 6, p. 13-26, 2005.

DOMÍNGUEZ, E. \& FERNÁNDEZ, H. R. Macroinvertebrados Bentónicos Sudamericanos. Sistemática y Biología. Tucumán, Fundación Miguel Lillo, 2009.

FERREIRA-PERUQUETTI, P. S. \& GESSNER, A. A. F. Comunidade de Odonata (Insecta) em áreas naturais do Cerrado e monocultura no nordeste do estado de São Paulo, Brasil: relação entre o uso do solo e a riqueza faunística. Revista Brasileira de Zoologia, Curitiba v. 20, p. 219-224, 2003.

FERREIRA-PERUQUETTI, P. S. \& De MARCO, P. Efeito da alteração ambiental sobre comunidades de odonatas em riachos de Mata Atlântica de Minas Gerais, Brasil. Revista Brasileira de Zoologia, Curitiba, v. 19, p. 317- 327, 2002.

FLOSS, E. C. S. et al. Diversity of non-biting midge larvae assemblages in the Jacuí River basin, Brazil. Journal of Insect Science, Tucson, v. 12, p.1-33, 2012.

GARRISON, R., VON ELLENRIEDER, N. \& LOUTON, J. Dragonfly Genera of the New World. An illustrated and annotated key to the Anisoptera. Baltimore, John Hopkins, 2006.

GOTELLI, N. J. \& COLWELL R. K. Quantifying biodiversity: procedures and pitfalls in the measurement and comparison of species richness. Ecology Letters, Oxford, v. 4, p. 379-391, 2001.

HECKMAN, C. W. Encyclopedia of South American Aquatic Insects: Odonata - Zygoptera. Illustrated Keys to Known Families, Genera, and Species in South America. Washington. Springer, 2008.

HUNDERTMARCK, I. S. \& MIORIN, V. M. F. Classificação das zonas agroecológicas na subbacia do rio Ibicuí-Mirim: adequação das culturas ao tipo de solo - RS; Geografia: Ensino e Pesquisa, Santa Maria, v. 11, n. 1, p. 9-15, 2001.

IBGE. RADAMBRASIL, Levantamento de Recursos naturais. Rio de Janeiro. IBGE, 1986.

IUCN. Red List of threatened species. 2008. Disponível em: $<w w w . i u c n r e d l i s t . o r g>$. Acesso em 15.10.2012.
JUEN, L., CABETTE, H. R. S. \& De MARCO, P. Odonate assemblage structure in relation to basin and aquatic habitat structure in Pantanal wetlands. Hydrobiologia, Den Haag, v. 579, p. 125-134, 2007.

KALKMAN, V. J. et al. Global diversity of dragonflies (Odonata) in freshwater. Hydrobiologia, Den Haag, v. 595, p. 351-363, 2008.

KIKUCHI, R. M. \& UIEDA, V. S. Composição e distribuição dos macroinvertebrados em diferentes substratos de fundo de um riacho no município de Itatinga, São Paulo, Brasil. Entomología y Vectores, Rio de Janeiro, v. 12, n. 2, p. 193-231, 2005.

MACHADO, A. B. M., MESQUITA, H. G. \& MACHADO, P. A. R. Contribuição ao conhecimento dos odonatos da estação ecológica de Maracá - Roraima. Acta Amazonica, Manaus, v. 21, p. 159-173, 1991.

MALUF, J. R. T. Nova classificação climática do estado do Rio Grande do Sul. Revista Brasileira de Agrometeorologia, Santa Maria, v. 8, p. 141-150, 2000.

MALTCHIK, L. et al. Responses of odonate communities to environmental factors in southern Brazil wetlands. Journal of the Kansas Entomological Society, Lawrence, v. 83, p. 208-220, 2010.

McKIE, B. G., PEARSON, R. G. \& CRANSTON, P. $\mathrm{S}$. Does biogeographical history matter? Diversity and distribution of lotic midges (Diptera: Chironomidae) in the Australian Wet Tropics. Austral Ecology, Carlton, v. 30, p. 1-13, 2005.

PAIVA, J. B. D. et al. Quantificação da demanda de água na bacia do rio Ibicuí - RS. Revista Brasileira de Recursos Hídricos, Porto Alegre v. 5, p. 93-111, 2000.

PEREIRA, P. R. B. et al. Contribuição à geografia física do município de Santa Maria: unidades de paisagem. Geografia Ensino e Pesquisa, Santa Maria, v. 3, p. 7-68, 1989.

QUADROS, F. L. F. \& PILLAR, V. P. Transições floresta - campo no Rio Grande do Sul. Fitogeografia do Sul da América. Ciência \& Ambiente, Santa Maria, v. 24, p. 109-118, 2002. 
RAMBO, S. J. B. A Fisionomia do Rio Grande do Sul: ensaio de monografia natural. São Leopoldo, Unisinos, 1994.

RODRÍGUEZ-CAPÍTULO, A. Los Odonata de la República Argentina (Insecta): Fauna de agua dulce de la República Argentina. La Plata. PROFADU (CONICET), 1992.

SAMWAYS, M. J. \& STEYTLER, N. S. Dragonfly (Odonata) distribution pattern on urban and forest landscapes, and recommendations for riparian management. Biological Conservation, Barking, v.78, p.279-288, 1996.

SOUZA, L. O. I., COSTA, J. M. \& OLDRINI, B. B. 2007. Odonata. Guia on-line: Identificação de larvas de Insetos Aquáticos do Estado de São Paulo. Froehlich, C.G. (ORG.). Disponível em: $<$ http://sites.ffclrp.usp.br/aguadoce/Guia_online>. Acesso em: 01.09.2012.

TEIXEIRA, M. C. Contribuição para o conhecimento da fauna odonatológica do Rio Grande do Sul. Arquivos do Museu Nacional, Rio de Janeiro, v. 54, p. 17-24, 1971.

VIEIRA, E. F. Rio Grande do Sul: Geografia física e vegetação. Porto Alegre. Sagra, 1984. 